\title{
Una invitación al pluralismo legal
}

\author{
Manuel José Prieto Montt*
}

\begin{abstract}
RESUMEN
La idea de pluralismo legal (o jurídico) está escasamente representada en la literatura en español. Motivado por ello, el presente trabajo es una invitación para que el lector se familiarice con la genealogía y principales elementos del enfoque aportado por esta idea. Para tal propósito, en primer lugar, se discutirá la ideología del centralismo legal como principal blanco de ataque del pluralismo legal, luego, en segundo lugar, se desarrollará la alternativa ofrecida por el pluralismo legal, destacándose sus diversas manifestaciones, evolución y críticas.
\end{abstract}

Pluralismo legal - centralismo legal - antropología jurídica

\section{An invitation to legal pluralism}

\begin{abstract}
The idea of legal (or juridical) pluralism is poorly represented in the Spanish speaking literature. Motivated by this fact, this work is an invitation for the reader to become familiar with the idea of legal pluralism, its genealogy and its main elements. For that purpose, firstly, it discusses the ideology of the legal centralism as the main target of the legal pluralism. Secondly, it develops the alternative that legal pluralism offers, highlighting its evolution and its various manifestation and criticisms.
\end{abstract}

Legal pluralism - legal centralism - legal anthropology

* Abogado y Magíster en Asentamientos Humanos y Medio Ambiente. Investigador asociado al Programa de Derecho y Política Ambiental, Escuela de Derecho, Universidad Diego Portales, Santiago, Chile. Correo electrónico: mjpm@email.arizona.edu. Dirección postal: Harvill Building, Box \#2 Tucson, AZ, 857210076, USA. Agradezco los comentarios de Carl Bauer y Thérèse de Vet a este trabajo, quienes quedan libres de toda responsabilidad por su contenido.

Artículo recibido el 30 de marzo de 2012 y aceptado para su publicación por el Comité Editorial el 25 de mayo de 2012. 


\section{INTRODUCCIÓN}

A ndré Singer, en su documental Brujería entre los $A z a n d e^{1}$, narra cómo un hombre y una mujer, luego de haber sido sorprendidos en una situación comprometedora, fueron acusados por sus respectivas parejas de haber incurrido en adulterio. La acusación se interpone ante el jefe de la comunidad quien, sentado en un escritorio y utilizando vestimenta de militar europeo, llama a las partes a comparecer a juicio. Luego de formulados los cargos, y negados éstos por parte de los acusados, el juez decide que el caso sea resuelto por un oráculo llamado benge. El juez llama al operador del oráculo, un hombre quien forzará a un polluelo a beber una pócima ritual. Al mismo tiempo que el ave bebe la sustancia, el operador repite numerosas veces "si los acusados yacieron juntos deja que el ave muera". A los pocos segundos el ave muere y, con ello, el crimen ha quedado comprobado. El juez sanciona a los acusados con trabajo comunitario y les exige un pago equivalente al costo de operación del oráculo. Tal como describe $C_{h a s e}^{2}$, las partes podrán apelar a los oráculos operados por príncipes superiores al jefe de la tribu.

Stephen Lansing en su libro Un Orden Perfecto ${ }^{3}$ analiza los modelos tradicionales de agricultura de arroz en Bali, Indonesia. En su relato, Lansing explora el cómo diversos grupos de agricultores balineses y sacerdotes se reúnen regularmente en templos del agua con el propósito de tomar decisiones y resolver la forma de administrar sus sistemas de irrigación. A pesar de que no existe un orden jerárquico, reglas estatales, ni un poder central, los agricultores de arroz han logrado coordinar sus decisiones por medio de ritos del agua desarrollados dentro de templos. Tras varios años de trabajo de campo, Lansing concluye la existencia de un complejo sistema de autoorganización cooperativa, por medio del cual los agricultores resuelven la coordinación entre los usuarios aguas arriba y aguas abajo, las disputas por los derechos de agua y el manejo de pestes.

El proceso de globalización económica ha impulsado el desarrollo de una serie de órdenes regulatorios que se construyen al margen de la ley del Estado. En este sentido, Tamanaha ${ }^{4}$ destaca cómo las transacciones comerciales internacionales, de manera creciente, se llevan a efecto dentro de marcos regulatorios, los cuales no necesariamente emanan de algún régimen internacional legal o desde algún Estado-nación específico. La obligatoriedad de dichos marcos emana de ciertas prácticas internacionales, contratos comerciales, estándares convencionales y usos y costumbres comerciales. Como contrapartida a esta situación, diversas organizaciones no gubernamentales presionan a las compañías trasnacionales para adoptar mejores prácticas (e.g., laborales, ambientales).

\footnotetext{
${ }^{1}$ Singer, A., Witchcraft among the Azande, Granada Television International (Filmmakers Library), 1982.

${ }^{2}$ Chase, O. G., Law, Culture, and Ritual: Disputing Systems in Cross-Cultural Context, New York University Press, New York, 2005.

${ }^{3}$ Lansing, J. S, Perfect Order: Recognizing Complexity in Bali, Princeton University Press, Princeton, N.J., 2006.

${ }^{4}$ Tamanaha, B. Z., "Understanding Legal Pluralism: Past to Present, Local to Global", en The Sydney Law Review, vol. 30, N³, 2008, pp. 375-411.
} 
En conjunto, estas prácticas dan cuenta y reconocen la existencia de múltiples órdenes regulatorios que operan a escala global y al margen de los estados-naciones.

Considerando estos ejemplos, surge la interrogante de si es posible afirmar que el juicio de los Azande, las decisiones adoptadas en los templos del agua y los órdenes regulatorios de las prácticas comerciales internacionales son calificables de derecho. La respuesta a esta pregunta dependerá de la posición teórica que se adopte a la hora de observar el fenómeno de la convivencia entre distintos sistemas o prácticas normativas que, operando como sistemas de control social, cohabitan dentro de un mismo espacio político.

En primer lugar, es posible considerar una respuesta desde la ideología dominante del centralismo legal. Como puede desprenderse de Griffiths ${ }^{5}$, esta ideología restringe el dominio legal al "derecho del Estado" o "al derecho oficial”. Para esta ideología sólo son derecho aquellas normas de conducta sancionadas por el Estado, siguiendo los principios de las democracias modernas, y que son reconocidas como tales por los discursos académicos que dominan la producción y reproducción del conocimiento jurídico. Estas normas, por su parte, se entenderán como el único sistema de control social que aparece como naturalmente legitimado ante los ojos del sentido común. En breve, para el centralismo legal, derecho y Estado son sinónimos.

Desde luego, ante el prisma de la ideología del centralismo legal, las prácticas seguidas por el juicio de los Azande y en los templos del agua en Bali serán presentadas, respectivamente, ya sea como simple rito, religión, tradición o costumbre. Si a esta descripción se le suma un modelo lineal de análisis evolutivo, además serán calificadas de premodernas, esotéricas, primitivas, atrasadas, paganas, mágicas o prejurídicas. Las prácticas comerciales internacionales descritas por Tamanaha serán analizadas como mera costumbre comercial que informa al derecho oficial.

Al contrario de la perspectiva del centralismo legal, ante la luz de las actuales perspectivas aportadas por los estudios críticos del derecho (Critical Legal Studies) y la antropología jurídica, se advierte que la visión del centralismo legal dista mucho de ser una perspectiva natural u obvia. No es necesario detallar ni justificar en este trabajo los distintos aportes críticos que, construidos desde una perspectiva anticolonial, de economía política, o postestructuralista, rechazan la naturalidad del centralismo legal. Por el momento basta afirmar que cualquier análisis sociolegal o de antropología jurídica, que tome en serio la teoría social crítica, advertirá el carácter colonialista, eurocéntrico, burgués y discursivo del proyecto defendido por el centralismo legal. Muchas de estas críticas se articulan bajo la perspectiva del pluralismo legal (o pluralismo jurídico).

Luego de revisar la base de datos de $\mathrm{SciELO}^{6}$ (y en menor grado otras fuentes) se ha advertido la escasa discusión y desarrollo en lengua española, tanto dentro de las publicaciones de derecho como de antropología, de la idea de pluralismo legal. Los

${ }^{5}$ Griffiths, J., "What is legal pluralism?", en Journal of Legal Pluralism and Unofficial Law, vol. 24, 1986, pp. $1-55$.

${ }^{6}$ Scientific Electronic Library Online (http://www.scielo.org/). 
escasos trabajos existentes se restringen predominantemente a discutir el tema de la apertura del derecho positivo occidental al reconocimiento del derecho indígena ${ }^{7}$. Otros trabajos analizan nuevas perspectivas en la autonomía de la voluntad ${ }^{8}$ o se restringen a casos específicos como la gestión del agua en las zonas andinas?.

Sin desmerecer el gran aporte de estos trabajos, ellos presentan una escasa discusión o reconocimiento de la extensa e importante literatura publicada en inglés sobre pluralismo legal y su desarrollo. Ante ello, y considerando la necesidad de enriquecer los debates y análisis de los estudios sociolegales en Chile, en lo que sigue se pretende llenar este vacío mediante el desarrollo de una breve introducción/genealogía del pluralismo legal. Para tales efectos, en primer lugar se desarrollará la perspectiva del centralismo legal y su configuración en tanto ideología. En segundo lugar, como respuesta crítica al centralismo legal se presentará la alternativa ofrecida por el pluralismo legal, destacándose sus diversas manifestaciones, evolución y críticas.

Si bien es cierto el presente trabajo no pretende en caso alguno cubrir una revisión completa de la exégesis y discusiones relativas al pluralismo legal, sí presenta un relato suficiente y una bibliografía adecuada como para introducir e invitar al lector a un estudio más profundo ${ }^{10}$.

\section{LA IDEOLOGÍA DEL CENTRALISMO LEGAL}

El pluralismo legal nace como una reacción crítica a la idea del derecho como centrado única y exclusivamente en el Estado. En términos de Galanter ${ }^{11}$, el pluralismo legal es un rechazo al "centralismo legal”. Esto es, la idea de que el Estado tiene el monopolio exclusivo de la producción del derecho, lo que equivale a decir que el derecho es igual a "derecho del Estado" o, en términos de Fitzpatrick, que el derecho

7 Ver: Castro, M., “Agua, derechos y cultura en los Andes del norte de Chile. Un enfoque desde la antropología jurídica”, en Chungara, vol. 29, N 1, 1997, pp. 63-80; Bernard, B., "Interpretación postmoderna del pluralismo legal como fundamento para una nueva Teoría del Derecho", en Frónesis, vol. 13, N 1, 2006, pp. 1315-6268; García, L., "Pluralismo Legal y Derecho Indígena”, en Frónesis, vol. 13, N 1, 2006, pp. 13156268. 2006; Carmona, C., “Derecho y Violencia: Reescrituras en torno al pluralismo jurídico”, en Revista de derecho (Valdivia), vol. 22, N², diciembre 2009, pp. 9-26; Castro, M. (ed.), Actas del XII Congreso Internacional. Derecho Consuetudinario y Pluralismo Legal: Desafíos del Tercer Milenio, Universidad de Chile, Santiago, Chile, 2000.

8 Perrin, J. F., "La autonomía de la voluntad y el pluralismo jurídico en nuestros días”, en Sociologías, $\mathrm{N}^{\circ} 13,2005$, pp. 162-178.

${ }^{9}$ En este sentido se destaca el trabajo desarrollado por Walir (Water, Law and Indigenous Rights). Dentro de sus publicaciones ver e.g., Boelens, R. A. (ed.)., Pluralismo Legal, Reformas Hídricas y Políticas de Reconocimiento. Walir, 2007.

${ }^{10}$ Para una revisión genealógica del pluralismo legal y discusiones asociadas se recomienda ver: Merry, S. E. "Legal Pluralism", en Law E Society Review. vol. 22. N 5, 1988, pp. 869-892; Tamanaha, B. Z., op. cit., n. 4.; Vanderlinden, J., "Return to legal pluralism: twenty years later", en Journal of Legal Pluralism and Unofficial Law; $\mathrm{N}^{\circ} 28,1989$, pp. 149-157.

${ }^{11}$ Galanter, M., "Justice in Many Rooms: Courts, Private Ordering and Indigenous Law”, en Journal of Legal Pluralism, vol. $19 \mathrm{~N}^{\circ} 1,1981$, pp. 1-47. 
es un tipo de acción del Estado ${ }^{12}$. Esta relación sinónima entre Estado y derecho es el contenido axiomático de la ideología del centralismo legal, contra el cual emergerá el pluralismo legal.

El hecho de que la sinonimia entre Estado y derecho sea una idea tan aceptada y difundida, y casi parte del sentido común, da cuenta del éxito del proceso de construcción del Estado moderno y la hegemonía de las ideas sobre la cual dicha evolución se fue desarrollando. Como elementos clave dentro de este proceso, Tamanaha ${ }^{13}$ analiza y destaca cómo durante los siglos XVII y XVIII se consagra la radical separación entre el dominio de lo público y lo privado. Aquí, costumbre, prácticas locales y religión se reducirán a un segundo ámbito, cambiando de este modo su estatus histórico de ley autónoma. Un segundo aspecto clave será la transformación experimentada por el rol del gobierno y del derecho. Estos, como parte del proceso liberal-moderno, ya no serán entendidos como un reflejo permanente de la costumbre o de algún principio de una supuesta naturaleza, sino que se configurarán como instrumentos racionales para alcanzar objetivos sociales compartidos por medio de un aparataje institucionalizado de poder. Aplicando la terminología de Foucault ${ }^{14}$, se puede decir que el centralismo legal transforma al aparataje del Estado en el único instrumento de verificación legal. Utilizando la conceptualización de Althusser, el derecho es reducido exclusivamente a los aparatos represivos del Estado ${ }^{15}$.

Ahora, ¿qué quiere decir que la idea de Estado, entendido como única fuente de verificación y creación del derecho, sea considerada como una ideología?

El primero en definir la ideología del centralismo legal es Griffiths, quien sostiene que el centralismo legal se basa en que:

el derecho es y debe ser el derecho del estado, uniforme para todas las personas, exclusivo para todos otros derechos, y administrado por un conjunto único de instituciones estatales. En la medida de que existan otros ordenamientos normativos menores, como la iglesia, la familia, las asociaciones voluntarias y las organizaciones económicas, ellos deberán estar subordinados, y de hecho lo están, al derecho y a las instituciones del estado ${ }^{16}$.

Ahora bien, Griffiths enuncia que esta idea de derecho como sinónimo de Estado es una ideología en la medida que su contenido substantivo se asume como algo a priori y entendido como una suposición natural e incuestionable.

\footnotetext{
${ }^{12}$ Fitzpatrick, P., Law and State in New Guinea, Academic Press, London, 1981.

${ }^{13}$ Ver detalles en: Tamanaha, B.Z., op. cit., n. 4.

${ }^{14}$ Foucault, M., Nacimiento de la Biopolítica: Curso en El College de France 1978-1979, Fondo de Cultura Económica, Argentina, 2007.

15 Althusser, L., Ideología y aparatos ideológicos del Estado, Quinto Sol, México, D. F., 1985.

${ }^{16}$ Griffiths J., op. cit., n. 5, p. 3. [La traducción es mía].
} 
Si se analiza el centralismo legal a la luz de la conceptualización más clásica del concepto de ideología, la de $\mathrm{Marx}^{17}$, se advierte su carácter ideológico en la medida de que su articulación supone una tergiversación de la realidad por medio de la negación del derecho no-estatal. A ello habría que agregar el hecho de que dicha tergiversación es producto de una reflexión consciente por parte de la clase dominante. A través de esta negación consciente, la clase dominante utilizará la ideología del centralismo legal con el propósito de presentar sus intereses como universales y, por consiguiente, generales de la sociedad toda ${ }^{18}$. Siguiendo esta noción, el derecho oficial opera como una creatura ideológica que permite a la clase dominante, en primer lugar, organizar la sociedad bajo sus propios intereses, en segundo lugar, forzar la materialización de esos intereses por medio de los aparatos represivos del Estado y, por último, presentar la sinonimia Estado-derecho como universal, natural, objetiva y ahistórica.

Aplicando el concepto de ideología planteado por Ž ižek ${ }^{19}$, el centralismo legal mediará la relación entre el derecho visible y el derecho imaginable bajo una dinámica en la que un derecho no-estatal aparece como algo imposible, inimaginable, y fuera de nuestro campo conceptual. Es más, el derecho estatal representará un orden simbólico naturalizado (en el sentido de Barthes) ${ }^{20}$, el cual no admitirá cuestionamiento alguno, ya sea de tipo histórico o político.

Desde luego, hay quienes insistirán en que el derecho necesariamente nace como una forma de control social monopolizada por una única institución soberana (i.e., quienes siguen a Weber), y todo lo que queda fuera de dicha forma de control es otra cosa, pero jamás podrá ser calificable de derecho. Para esta perspectiva, el centralismo legal no es erróneo, no es ideológico, ni mucho menos refleja los intereses de la clase dominante. El centralismo legal, simplemente refleja la verdad de que el Estado es el amo y señor de la producción jurídica. Tal vez la doctrina del centralismo legal sea la correcta y, por consiguiente, sólo el Estado (e.g., Weber) o alguna regla de reconocimiento formal (e.g., Hart) sean las únicas fuentes válidas del derecho. Sin embargo, cuando el concepto de ideología es ampliado, esta potencial veracidad no anula el carácter ideológico del centralismo legal.

De acuerdo con Žižek ${ }^{21}$, el contenido de la ideología no necesariamente debe ser falso (como lo sostendría, por ejemplo, el marxismo clásico). En su opinión, el contenido ideológico puede ser o no verdadero, ello es irrelevante para cualquier proyecto antiideológico. Lo relevante aquí es destacar cómo los contenidos de la ideología (sean o no verdaderos) forman parte de las relaciones de poder. En este sentido, sin importar si la

\footnotetext{
${ }^{17}$ Para una revisión del concepto de ideología en Marx ver: Larraine, J., El Concepto de Ideología, LOM, Santiago, Chile, 2007.

${ }^{18}$ Ver la formulación de ideología planteada por Harvey, D., "The Marxian Theory of the State”, en Antipode, vol. 8, N², 1976, pp. 80-89.

19 Žižek, S., "El espectro de la ideología”, en Žižek, S. (ed.), Ideología un mapa de la cuestión, Fondo de Cultura Económica, Buenos Aires, Argentina, 2003, pp. 7-42.

${ }^{20}$ Barthes, R., Mitologías, Siglo XXI, Buenos Aires, Argentina, 2002.

${ }^{21}$ Žižek, S., op. cit., n. 18.
} 
idea de derecho guarda o no una relación sinonímica con el Estado, se estará en frente a la ideología del centralismo legal mientras esa relación sinonímica sea funcional a alguna forma de dominación, poder o explotación social. Dicho de otro modo, puede ser que quienes defiendan al Estado como única fuente de producción jurídica estén en lo correcto, sin embargo, mientras su defensa del derecho único y monopolizado por el Estado esté al servicio del poder ejercido por algunos sobre otros, es irrelevante la veracidad de sus afirmaciones para ser calificadas o no de ideológicas.

Ante la ideología del centralismo legal, y como un proyecto que apunta a develar las relaciones de poder ocultas ya sea tras la sinonimia entre Estado y derecho, o tras la idea de derecho único dentro de un mismo espacio político, surge la perspectiva del pluralismo legal.

\section{LA ALTERNATIVA DEL PLURALISMO LEGAL}

El problema de la relación entre cultura, moral y Estado ha sido objeto de un viejo debate entre las tradiciones liberales y comunitaristas. En el eje de este debate están el rol y posición del derecho frente al fenómeno de la pluralidad o diversidad cultural. Sin embargo, desde ambos polos en debate se mantiene y reproduce la fe en el Estado como entidad unificada y fuente de un orden jerarquizado y sistemático de normas jurídicas. En este sentido, no cabe confundir el pluralismo legal (el tema convocado en este trabajo) con el debate sobre el reconocimiento de la diversidad cultural por parte de los Estados y del proyecto defendido por las democracias liberales. Por el contrario, el pluralismo legal se desmarca de este ya antiguo debate y, cuestionando al Estado como fuente unitaria del derecho, usa el concepto de "pluralismo" con el propósito de, en primer término, rechazar la ideología del centralismo legal para luego, en segundo término, constatar la coexistencia de múltiples órdenes, prácticas o sistemas legales (estatales o no) dentro de un mismo espacio político o campo social ${ }^{22}$. En este sentido, en una de las obras claves del pluralismo legal, Griffiths rechaza la idea de que:

el derecho es un sistema exclusivo, sistemático, jerárquico de proposiciones normativas, que pueden ser miradas ya sea desde arriba hacia abajo como dependientes del comando soberano (Bodin, 1576; Hobbes, 1851; Austin, 1832) o desde abajo hacia arriba derivando su validez desde capas más generales de normas hasta que alcanzan la última norma(s) (Kelsen, 1949; Hart, 1961) ${ }^{23}$.

Luego de este rechazo al centralismo jurídico, el pluralismo legal constatará la pluralidad de órdenes legales como un hecho empírico. Esto supone la coexistencia de

${ }^{22}$ Ver e.g., Pospisil, L., The Anthropology of Law: A Comparative Theory of Law. Harper and Row, New York, 1971; Griffiths, J., op. cit., n. 5; Santos, B., Law: A Map of Misreading. "Toward a Postmodern Conception of Law", en Journal of Law and Society. vol. 14, N³, pp. 279-302; Merry, S. E., op. cit., n. 10.

${ }^{23}$ Griffiths, J., op. cit., n. 5, p. 3. [La traducción es mía]. 
múltiples órdenes legales cuya existencia no depende de que si emanen o no de fuentes oficiales de carácter estatal. Mediante esta constatación, el pluralismo jurídico no buscará resolver problemas de moralidad, o de inconsistencia legal, con el propósito de salvaguardar la existencia del derecho como un sistema exclusivo, único, unificado y consistente. Por el contrario, el pluralismo jurídico desmitifica esta visión con el propósito de romper jerarquías y develar las relaciones de poder dentro de una pluralidad de derechos como un hecho empírico.

\section{LAS RAÍCES ETNOGRÁFICAS DEL PLURALISMO LEGAL}

Los estudios de antropología jurídica, cuyas preguntas fueron derivando en lo que hoy se conoce como pluralismo legal, corresponden a aquellas etnografías legales llevadas a cabo en contextos disímiles a las prácticas jurídicas de Europa y Estados Unidos ${ }^{24}$. Las preguntas originales que movilizaron estas etnografías se restringían a develar el cómo es posible la existencia de un orden social sin la presencia del Estado como entidad monopolizadora de la producción del derecho. Desde luego, este problema implica una reflexión mayor en torno a las preguntas de qué prácticas sociales pueden ser consideradas como derecho y cómo se distingue el derecho de otros sistemas de control social.

Durante este estadio de la antropología jurídica se destacan diversas etnografías relevantes. En este sentido, uno de los trabajos pioneros es el desarrollado por Malinowski ${ }^{25}$ en las islas Trobriand. Su problema principal es intentar dilucidar el problema del orden social y su reproducción en un contexto de ausencia de instituciones formales, como las propias de un Estado moderno (e.g., policía, cortes, poder legislativo, derecho escrito). Por su parte, Gluckman ${ }^{26}$, tras estudiar los procesos de resolución de conflicto seguidos por los Barotse o Lozi del norte de lo que fue Rodesia ${ }^{27}$, concluye que sus normas eran únicas a su grupo social, sin embargo, el razonamiento sobre las cuales se construían era parte de una lógica jurídica universal. Ello, de acuerdo con Moore ${ }^{28}$, daría cuenta de que Gluckman "buscaba mostrar que los sistemas y prácticas legales de los indígenas africanos eran igualmente racionales, en un sentido weberiano, que los occidentales" 29 . Dentro de este mismo periodo, otro trabajo, el de Bohannan ${ }^{30}$, rechazará el universalismo jurídico de Gluckman. Basado en sus estudios sobre los Tiv de Nigeria, Bohannan

${ }^{24}$ Moore, S. F. Law and Anthropology: A Reader, Blackwell, Malden, MA. 2005.

${ }^{25}$ Malinowski, B., Crime and Custom in Savage Society, Harcourt, Brace and Co., New York, 1932.

${ }^{26}$ Gluckman, M., The judicial process among the Barotse of Northern Rbodesia, Manchester U.P., Manchester, 1967.

${ }^{27}$ Los Barotse o Lozi son un grupo étnico que habita predominantemente la zona oeste de lo que hoy es Zambia.

${ }^{28}$ Moore, S. F., "Certainties Undone: Fifty Turbulent Years of Legal Anthropology, 1949-1999”, en Journal of the Royal Anthropological Institute, vol. 7, N 1, 2001, pp. 98-116.

${ }^{29}$ Id., p. 98. [La traducción es mía].

${ }^{30}$ Bohannan, P., Justice and Judgment Among the Tiv, International African Institute by Oxford University Press, London, New York, 1957. 
se aleja de perspectivas de esencialismo legal, destacando que el derecho emerge de las particularidades únicas de cada cultura, y cualquier intento de universalización implica una traducción ${ }^{31} \mathrm{y}$, por ende, una distorsión ${ }^{32}$.

Otras obras clásicas, dentro de esta línea de trabajo, son: el estudio de EvansPritchard ${ }^{33}$ quien, sin estar especialmente preocupado por temas legales, en su trabajo sobre oráculos y brujería entre los $A z$ ande de la zona alta del Nilo explora las conexiones entre ritualidad como forma de resolución de conflicto ${ }^{34}$; el trabajo de Popsipil sobre los Kapauku de Nueva Guinea ${ }^{35}$; la investigación de Hoebel \& Llewellyn sobre los Cheyenne de las Grandes Llanuras de EE.UU. ${ }^{36}$; el trabajo de antropología visual de Marshall sobre los !Kung ${ }^{37}$.

Este periodo no está exento de discusiones y tensiones entre las perspectivas y conclusiones de las obras citadas. Ello, especialmente en lo referente a qué es lo que se entiende por derecho y, en relación a ello, las interrogantes que surgen de la posibilidad de que el etnógrafo reconstruya su caso de estudio a partir de las categorías jurídicas presentes en su lugar de origen ${ }^{38}$. Ahora bien, pese a los desencuentros teóricos, el principal elemento rescatable de este estadio, el cual derivará en el futuro desarrollo del pluralismo legal, radica en el cuestionamiento del Estado como única fuente válida de derecho. Dicho de otro modo, las etnográficas legales de este período defienden la posibilidad de encontrar sistemas normativos de carácter legal en ausencia de un Estado. Sin embargo, a diferencia del futuro desarrollo del pluralismo legal, durante este período las etnografías no ponen énfasis en la relación dialéctica entre derecho colonial y las prácticas normativas indígenas o colonizadas. Si bien es cierto se reconoce de manera general que dichas prácticas sufren modificaciones y cambios tras los procesos de colonización, se siguen entendiendo como cuerpos normativos en paralelo o de carácter discreto.

${ }^{31}$ La idea de distorsión por medio de la traducción es tomada de Geertz, C. Local knowledge: Further essays in interpretive anthropology, Basic Books, New York, 1983.

32 Para detalles de la controversia Gluckman-Bohannan ver Von Benda-Beckmann, F. V., "Anthropology and Comparative Law" en Benda-Beckmann, K. V y Strijbosch, F. (eds.), Anthropology of Law in the Netherlands, Foris, Dordrecht, 1986, y Moore, S. F., op. cit., n. 27.

${ }^{33}$ Evans-Pritchard, E. E., Witchcraft, Oracles and Magic Among the Azande, Clarendon Press, Oxford, 1937.

${ }^{34}$ Para una revisión contemporánea del trabajo de Evans-Pritchard y una relación más explícita con los estudios legales, ver Chase, O. G., op. cit., n. 2. Ver también el trabajo de antropología visual de Singer, A., op. cit., n. 1 .

${ }^{35}$ Los Kapauku son un grupo ético papuano que habita en la zona del oeste-central de Nueva Guinea.

${ }^{36}$ Llewellyn, K. N. y Hoebel E. A., The Cheyenne Way: Conflict and Case Law in Primitive Jurisprudence, University of Oklahoma Press, Norman, 1941. Los Cheyenne son los indígenas originarios de la zona de América del Norte conocida como las Grandes Llanuras.

${ }^{37}$ Marshall, J., The meat fight. Watertown, MA: Documentary Educational Resources, 2007. El trabajo es original de 1974. Los !Kung o tribu de los Ju/'hoansi habitan las zonas de Botswana, Angola y Namibia.

38 Moore, op. cit., n. 23. 


\section{FundaCión y PLURALISMO LEGAL CLÁSICO}

Como advierten Tamanaha ${ }^{39}$ y Merry $^{40}$, el reconocimiento del pluralismo legal como enfoque disciplinario nace en la década de 1970 con los estudios de sociedades en contextos coloniales y postcoloniales, dando origen a lo que Merry describe como "pluralismo legal clásico" 41.

En esta etapa fundacional, el análisis se focaliza en comprender cuál es la dinámica de interacción entre diversos órdenes normativos, los cuales, pese a compartir un mismo espacio político, son radicalmente diferentes en términos culturales, estructurales y conceptuales. Ello, en la medida de que se manifiestan dentro de una situación de colonialismo o postcolonialismo. De allí que, como sostiene Schiff ${ }^{42}$, durante esta etapa el pluralismo legal pondrá énfasis en la coexistencia de sistemas legales diversos, imaginados como distintos y organizados de manera jerárquica.

Como se podrá apreciar, en esta etapa fundacional el objetivo será el comprender el fenómeno de las intersecciones jerárquicas entre el derecho de los colonizados (principalmente indígenas) por parte del derecho de los colonos (principalmente derecho europeo). Como destaca Merry ${ }^{43}$, aquí los actores serán, en primer lugar, naciones dotadas de un aparataje legal centralizado y codificado, las que intentarán imponer sus sistemas normativos sobre, en segundo lugar, sociedades en las cuales predominan prácticas normativas basadas en la oralidad y en la ausencia de formalidades para juzgar y castigar. En suma, como advierte Tamanaha ${ }^{44}$, durante este periodo los estudios del pluralismo legal se radican en comprender las relaciones de tensión, coexistencia, reconocimiento/ no-reconocimiento existente entre el derecho de los colonizados y el derecho de los colonizadores. Dentro de este contexto, alguno de los trabajos fundacionales de mayor relevancia son los de Pospisil y Hooker.

Pospisil $^{45}$ entenderá el pluralismo legal como un fenómeno de coexistencia entre diversos regímenes normativos e institucionales en conjunto con un derecho oficial estatal. Su descripción simplemente constata una pluralidad de normas e instituciones, las que, reclamando autonomía, conviven con el derecho de Estado, sin importar si éste los reconoce de manera oficial o no. De acuerdo a su descripción, en cada sociedad hay subgrupos que desarrollan sus propios sistemas legales, los cuales son posibles de diferenciar respecto del resto de los subgrupos que cohabitan dentro de una misma sociedad (e.g., familias, comunidades, organizaciones políticas). Por otro lado, Hooker ${ }^{46}$ niega

\footnotetext{
39 Tamanaha, B. Z., op. cit., n. 4.

${ }^{40}$ Merry, S. E., op. cit., n. 10.

${ }^{41} I d$.

42 Schiff, P., "The New Legal Pluralism”, en Annual Review of Law and Social Science, vol. 5, 2009, pp. 225-242.

${ }^{43}$ Merry, S. E., op. cit., n. 10.

44 Tamanaha, B. Z., op. cit., n. 4.

45 Pospisil, L., op. cit., n. 21.

46 Hooker, M. B. Legal Pluralism: An Introduction to Colonial and Neo-Colonial Laws. Clarendon Press,
} Oxford, 1975. 
el pluralismo legal como un fenómeno de coexistencia independiente entre subgrupos normativos. En su caso, tras años de estudio en Asia, África y Oriente Medio, él observa cómo los sistemas legales atraviesan fronteras culturales. Ello lo lleva a entender al pluralismo jurídico como una cuestión de incorporación/reconocimiento de las costumbres locales por parte del Estado central.

Uno de los aportes del pluralismo legal clásico a la antropología jurídica en general y a los estudios postcoloniales en particular es su cuestionamiento a las ideas de la costumbre como algo autónomo y partícipe de un proceso lineal de evolución jurídica.

En primer término, dentro del contexto colonial, la manera en que las costumbres locales se relacionaban con el derecho del Estado colonizador se efectuaba/efectúa, como destaca Tamanaha ${ }^{47}$, bajo tres estrategias: (a) mediante la codificación expresa de la costumbre, (b) mediante el reconocimiento judicial de la costumbre, o (c) mediante la creación de cortes especiales destinadas a resolver los conflictos en los que la costumbre fuese parte del asunto. Desde luego, estas tres alternativas daban/dan un gran poder de manipulación por parte del derecho estatal respecto al orden normativo local, limitando su incorporación en la medida en que se guardase compatibilidad con los intereses, ya sean económicos o morales del colonizador. Basado en ello, Merry ${ }^{48}$ presenta una detallada discusión de cómo lo que se conoce como costumbre, o derecho tradicional, es en realidad producto de un proceso de mitificación colonial, construido con el propósito de imponer el modelo de orden colonial y centralizado. Dentro de esta discusión es posible encontrar desde perspectivas que entienden a la costumbre como una mera adaptación o transformación por parte del colonizador, a enfoques más radicales, los cuales plantean a la costumbre como una nueva creación jurídica establecida por el poder colonial.

Si bien es cierto la idea dominante tras el derecho colonial radica en su imposición como instrumento de expansión capitalista, Merry ${ }^{49}$ problematiza y discute esta noción. Incorporando interesantes elementos al debate, en primer lugar, la autora da cuenta de que lo que se conoce como derecho de costumbre no fue universalmente impuesto de manera unilateral, sino también existió una acomodación por parte de las elites colonizadas del derecho foráneo. En este sentido, un excelente ejemplo es el caso presentado por Banner ${ }^{50}$ respecto de las reformas a la propiedad del suelo en Hawai. En este caso, las elites hawaianas, preparándose ante una inminente colonización, importaron y adaptaron un régimen de propiedad foráneo para resguardar sus propios intereses ante los potenciales colonizadores. En segundo lugar, otro elemento importante es la paradoja de cómo el derecho colonial no opera únicamente como un instrumento de dominación ni culturización, por el contrario, comunidades colonizadas también se hacen valer de

47 Tamanaha, B. Z., op. cit., n. 4.

48 Merry, S. E., op. cit., n. 10.

49 Merry, S. E., "Colonial and Postcolonial Law", en Sarat, A. Blackwell Companion to Law and Society. Blackwell Publishing, Oxford, 2004. pp. 569-588. Parte de esta discusión en id.

${ }^{50}$ Banner, S., "Preparing to be Colonized: Land Tenure and Legal Strategy in Nineteenth-Century Hawaii", en Law E Society Review, vol. 39, N², junio 2005, pp. 273-314. 
éste con el propósito de resistir al poder del colonizador ${ }^{51}$. Casos de estudio como el de Rodman ${ }^{52}$ también dan cuenta de qué manera tras el proceso colonizador en Vanuatu, y como respuesta a la ausencia de aparataje estatal colonial, los isleños desarrollaron innovadoras instituciones legales al margen del centralismo estatal.

Otro elemento clave dentro del pluralismo legal clásico es el rechazo a la costumbre como una etapa primitiva o precolonial que, dentro de un proceso más amplio de evolución jurídica, deriva en derecho positivo estatal. Subscribiendo a este rechazo, Diamond ${ }^{53}$ objeta cualquiera interpretación evolutiva del derecho y, por el contrario, afirma que el orden del derecho positivo estatal y el orden de la costumbre son completamente distintos. Es más, el derecho estatal, más que una cúspide dentro de supuesto proceso evolutivo, debería ser entendido más bien como una imposición jerárquica y antagónica que hace desaparecer la costumbre local y su forma de organizar el mundo.

\section{El NUEVO PLURALisMo LEGAL}

En su detallada genealogía sobre el pluralismo legal, Merry ${ }^{54}$ da cuenta de cómo los aportes del pluralismo legal clásico, concentrado en sociedades coloniales y postcoloniales, crean las raíces para el desarrollo de lo que ella denomina un nuevo pluralismo legal. En su opinión, el pluralismo legal clásico ha descrito las relaciones entre distintos órdenes normativos, ha delineado la dialéctica entre dichos órdenes y ha presentado a la costumbre como históricamente construida por los procesos de colonización. Estos aportes habrían sentado las bases etnográficas y teóricas necesarias para expandir los casos de estudio desde sociedades coloniales y postcoloniales a países industrializados, determinado así la emergencia de un "nuevo pluralismo legal".

Si bien es cierto tanto el pluralismo legal clásico como el nuevo problematizan la ideología del centralismo legal, la trayectoria en el estudio desde sociedades coloniales y postcoloniales hacia sociedades industrializadas permitirá un nuevo énfasis. El nuevo foco de estudio radica en las relaciones de poder experimentadas por una gama mayor de grupos que conviven en sociedad, de este modo:

el pluralismo legal se ha expandido desde un concepto que se refiere a las relaciones entre colonos y colonizados hacia relaciones entre grupos dominantes y subordinados, tales como religiosos, étnicos, minorías culturales, grupos de inmigrantes, y formas no oficiales de orden localizadas en redes sociales o instituciones ${ }^{55}$.

51 Merry, S. E., op. cit., n. 10.

52 Rodman, W. L., “A Law Unto Themselves": Legal Innovation in Ambae, Vanuatu”, en American Ethnologist, vol. 12, N 4, noviembre 1985), pp. 603-624.

53 Diamond, S., "The Rule of Law versus the Order of Custom”, en Black D. y Mileski, M. (eds.), The Social Organization of Law, Seminar Press, New York, 1973. pp. 318-344.

${ }^{54}$ Merry, S. E., op. cit., n. 10.

${ }^{55}$ Id. en p. 872. [La traducción es mía]. 
Lo relevante de la emergencia de este nuevo pluralismo radica en el hecho de que éste será un fenómeno presente en todas las sociedades, sin importar si experimentaron o experimentan dominación colonial. Esta trayectoria analítica determina el carácter de omnipresente del pluralismo legal, el cual se manifiesta a través de otras formas de regulación social que "se dibujan sobre los símbolos del derecho" 56 . Ello ha permitido una mayor amplitud disciplinaria, por cuanto, de ser el pluralismo clásico dominio exclusivo de la antropología jurídica, pasa a expandirse a otras disciplinas como parte de los estudios sociolegales ${ }^{57}$.

\subsection{Nuevos enfoques}

La amplitud en los casos de estudio del nuevo pluralismo legal también va acompañada con una amplitud en el enfoque. Ello, en el primer lugar, en la medida de que los distintos órdenes legales que conviven en el mismo espacio político ya no serán analizados como partícipes de una estructura jerárquica, dentro de la cual un tipo de sistema legal domina a los restantes. Por el contrario, siguiendo el trabajo de Moore ${ }^{58}$, la pluralidad jurídica estará compuesta por sistemas de orden social particulares a una pluralidad de campos sociales semiautónomos, los cuales se relacionan entre sí por medio de resistencia y autonomía, pero no bajo órdenes jerárquicos de dominación o reconocimiento formal. Siguiendo esta idea, el nuevo pluralismo legal verá los distintos regímenes legales partícipes de una relación dialéctica de mutua constitución. Ello, en la medida de que se abandonarán las explicaciones que muestran a sistemas legales dominantes influenciando unilateralmente a sistemas legales de menor jerarquía (i.e, el derecho colonial penetrando el derecho indígena). Por el contrario, el nuevo pluralismo legal pondrá énfasis en la bidireccionalidad, coconstitución, hibridización y en la dialéctica de la influencia ${ }^{59}$.

Dentro de esta línea, rechazando los órdenes jerárquicos y defendiendo la coconstitución de los distintos sistemas normativos dentro de un mismo espacio político, Santos presenta al "pluralismo legal como un concepto clave en una visión postmoderna del derecho" 60 . En su opinión, el pluralismo legal debe abandonar aquella visión del pluralismo legal clásico, en la que distintos órdenes legales coexisten dentro de un mismo espacio político como entidades discretas, diferenciables y en relación asimétrica. En vez de ello se hace necesario avanzar hacia una "concepción de diferentes espacios legales superimpuestos, interpenetrados y mezclados en nuestras mentes como en nuestras

${ }^{56}$ Id. n p. 874. [La traducción es mía].

57 Para una introducción y genealogía de los estudios "sociolegales" o de "Derecho y Sociedad" (Law E Society) ver: Silbey S. S. y Seron, C. "Profession, Science, and Culture: An Emergent Canon of Law and Society Research" en Sarat, A. Blackwell Companion to Law and Society. Blackwell Publishing, Oxford, 2004. pp. 30-59.

58 Moore, S. F., "Law and social change: the semi-autonomous social field as an appropriate subject of study”, en Law E Society Review, vol. 7, N 4. 1973, pp. 719-746.

${ }^{59}$ Ver e.g., Fitzpatrick, P. "Law and societies", en Osgoode Hall Law Journal, vol. 22. N 1, 1984, pp. 297.

${ }^{60}$ Santos, B., op. cit., n. 21. [La traducción es mía]. 
acciones" 61 . Ello configura el concepto de porosidad legal, la que supone "múltiples redes de órdenes legales forzándonos a constantes transiciones y traspasos" 62 . Sobre la base de estas ideas, Santos plantea el concepto de interlegality, el cual supone una "intersección de diferentes órdenes legales" 63 que llevan a comprender, bajo metáforas geográficas, el cómo distintos espacios legales operan al mismo tiempo, y dentro de mismos espacios políticos, a diferentes escalas, utilizando distintos símbolos y proyecciones ${ }^{64}$. Para ilustrar su propuesta, el autor presenta casos de estudio referentes a sistemas de administración de justicia en Cabo Verde y las favelas en Brasil.

Entre otros enfoques recientes, Teubner ${ }^{65}$ presenta al pluralismo legal desde la perspectiva autopoiética. ${ }^{66}$ En su opinión, el derecho experimentaría una pluralidad en la medida de que es un discurso que, dentro de su sistema de clausura, opera comunicativamente sobre la base de códigos binarios que establecen lo que es legal/ilegal. Bajo esta perspectiva toda acción comunicativa, provenga o no del Estado, que opere bajo esta dinámica, es calificable de derecho.

Criticando las nuevas propuestas de Santos y Teubner, como a su vez cualquier otro proyecto esencialista, Tamanaha ${ }^{67}$ presenta lo que él denomina una "aproximación convencional al pluralismo legal”. De acuerdo con ésta, el problema del pluralismo legal es que ha intentado generar ideas esenciales de lo que se entiende por derecho. Rechazando todo concepto unitario de derecho, el autor invita a definir al pluralismo legal de manera convencional. Aquí el derecho es entendido como una práctica social que, combinando significado y comportamiento, es considerada como derecho en la medida de que las personas que participan de determinado grupo social reconocen convencionalmente a dicha práctica como derecho. Toda vez que, dentro de un mismo espacio político, diversos grupos sociales reconozcan convencionalmente múltiples prácticas como derecho, se está en presencia de un pluralismo legal. Por medio de esta idea, Tamanaha llama a evitar formular por adelantado conceptos científicos de derecho, e invita a entender el derecho a través de las propias definiciones generadas por las personas quienes en su vida diaria hacen del derecho una práctica social.

${ }^{61} I d$., p. 298. [La traducción es mía].

${ }^{62} I d$.

${ }^{63} I d$.

${ }^{64}$ De allí que Santos sostenga la tesis de que el derecho debe ser entendido bajo la metáfora geográfica de un mapa. La principal idea en esta formulación es que el derecho, como los mapas, distorsionan la realidad por medio de la escala, la simbolización y la proyección. Al respecto ver $i d$.

65 Teubner, G., “The Two Faces of Janus: Rethinking Legal Pluralism”, en Cardozo Law Review, 1992, pp. 1443-62.

${ }^{66}$ Neologismo acuñado por la escuela de Santiago (Francisco Varela y Humberto Maturana) con el propósito

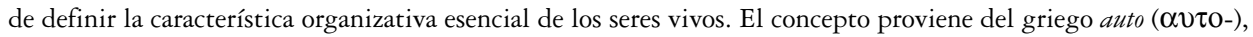

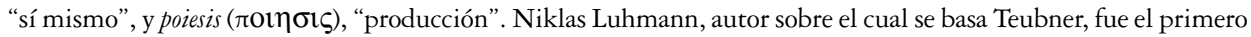
en utilizar dicho concepto desde la dogmática jurídica.

${ }^{67}$ Tamanaha, B. Z., “A Non-Essentialist Version of Legal Pluralism”, en Journal of Law and Society, vol. 27, $\mathrm{N}^{\circ} 2,2000$, pp. 296-321. 


\subsection{Pluralismo jurídico internacional y global}

El derecho internacional y el fenómeno de la globalización han sido un nicho importante para nuevos estudios en pluralismo legal. El hecho de que, en el campo globalizado del derecho internacional, convivan múltiples sistemas legales, exista una ausencia de órdenes jerárquicos y centralizados, y actores no-estatales adquieran cada vez más protagonismo, han sido motivaciones para elevar el estudio del pluralismo legal a la arena internacional.

El hecho de que el derecho internacional carezca de una autoridad central ha llevado a la antropología jurídica a plantearse la pregunta de ¿cómo funciona el derecho internacional?, ¿de dónde provienen esas leyes?, ¿cómo son aplicadas? y ¿de qué manera se relacionan con la pluralidad de sistemas normativos? Ante estas preguntas, el proyecto del pluralismo legal ha intentado entender la producción, operación, significancia del derecho internacional contemporáneo como parte de la pluralidad de sistemas normativos.

En este sentido, Merry ${ }^{68}$ muestra el cómo las preguntas que motivaron las primeras etnografías jurídicas, y que derivaron en el estudio del pluralismo legal, han servido para comprender los complejos ensamblajes entre normas, principios e instituciones que, en conjunto, constituyen el derecho internacional. A modo de ejemplo, la autora ilustra de qué manera mediante trabajos etnográficos es posible construir paralelos explicatorios entre el derecho internacional y los sistemas normativos mediante los cuales distintas villas se organizan entre sí en ausencia de poderes centrales y cortes que administren justicia.

Lo interesante del derecho internacional para el pluralismo legal radica en que representa una nueva capa o forma de derecho que, además de configurarse en sí mismo por pluralidades jurídicas, se suma a los múltiples sistemas legales que, interligados entre sí, operan a escala local. Diversos autores analizan este fenómeno.

Santos $^{69}$, utilizando metáforas cartográficas, ve que el pluralismo legal opera a pequeña, mediana y gran escala. Siguiendo esta idea, el derecho internacional representa un derecho que opera a pequeña escala, lo que supone una regulación de las acciones sociales de manera general, ignorando las particularidades y pluralidades de las escalas mayores $^{70}$. De Santos da cuenta de cómo la escala del derecho internacional no está compuesta de manera exclusiva por el "derecho oficial;" por el contrario, el fenómeno de la interlegalidad también operaría horizontalmente, incorporando de este modo cuerpos normativos informales, como la lex mercatoria.

${ }^{68}$ Merry, S. E., “Anthropology and International Law”, en Annual Review of Anthropology, vol. 35, 1996, pp. 99-116.

${ }^{69}$ Santos, B. S., op. cit., n. 21.

${ }^{70}$ En cartografía, la escala dice relación con el área de territorio proyectada y los detalles de la proyección. Al respecto, escalas mayores suponen una proyección más detallada de un área de territorio menor. Por el contrario, las escalas menores suponen un proyección menos detallada de un área de territorio mayor. A modo de ejemplo, el mapa del mundo es de una menor escala que el mapa de Chile. 
Examinando la fragmentación del derecho internacional, Burke-White ${ }^{71}$ advierte cómo el derecho internacional es internamente plural. De allí que el autor acuña el concepto de "pluralismo legal internacional”. En su opinión, el aplicar el foco del pluralismo legal ayudaría a evitar que la actual fragmentación del derecho signifique un riesgo para las instituciones, reglas y prácticas del derecho internacional.

Recientemente, Michaels ${ }^{72}$ analiza detalladamente de qué manera el fenómeno de la globalización implica la existencia de un pluralismo legal compuesto por una coexistencia entre el derecho doméstico con otros órdenes legales. En su trabajo discute la forma en que el pluralismo legal se relaciona con la globalización del derecho y, al mismo tiempo, cómo dicha globalización hace uso y existe a través del pluralismo legal. En esta misma línea, Berman ${ }^{73}$ llama a utilizar al pluralismo legal como una perspectiva para reenmarcar los viejos debates conceptuales para, de este modo, comprender y manejar los nuevos espacios legales y culturales que, producto de la globalización, se hibridizan.

Es notorio el hecho de que el derecho internacional, al ser de por sí plural, invite a un análisis desde el pluralismo legal. Además de esta motivación, Tamanaha ${ }^{74}$ advierte otros cuatro temas que demandan una perspectiva de pluralismo legal respecto del derecho internacional, estos son: internacionalización de los derechos humanos, la expansión de derecho internacional no-oficial, la proliferación de redes transgubernamentales y el movimiento global de personas.

\section{RECONECTANDO EL NUEVO PLURALISMO LEGAL CON SUS ORÍGENES: EL DERECHO COMO RITUAL}

Desde luego, dentro del nuevo pluralismo legal, los estudios de sociedades no industriales no han sido abandonados. Por el contrario, nuevos estudios en antropología jurídica han impulsado interesantes comparaciones que cuestionan los criterios por medio de los cuales se construyen las distinciones categóricas entre el derecho oficial y lo que está fuera de éste. Tal como afirma Merry, "claramente hay muchas diferencias entre el orden social de las villas y del mundo, pero también hay similitudes"75. Intentando trazar estas similitudes, el reciente trabajo de $\mathrm{Just}^{76}$ presenta una interesante reflexión.

Basado en sus investigaciones sobre los modelos de resolución de conflictos adoptados tanto por los Dou Donggo de Indonesia como por sociedades industriales (i.e., Estados Unidos y Gran Bretaña), Just rechaza toda distinción entre ritualidad y

${ }^{71}$ Burke-White, W. W., "International Legal Pluralism”, en Michigan Journal of International Law. vol. 25, No 4, 2004, pp. 963-979.

72 Michaels, R., “Global Legal Pluralism”, en Annual Review of Law E Social Science, vol. 5, 2009, pp. 243-62.

${ }^{73}$ Berman, P. S. "The New Legal Pluralism”, en Annual Review of Law and Social Science, vol. 5, 2009, pp. 225-242.

74 Tamanaha, B.Z., op. cit., n. 4.

${ }^{75}$ Merry, op. cit., n. 68, p. 101. [La traducción es mía].

76 Just, P., "Law, Ritual and Order", en Benda-Beckmann, K. V. y Pirie, F. (eds), Order and disorder: Anthropological perspectives, Berghahn Books, New York, 2007. 
derecho. La tesis principal del autor es que las propiedades presentadas por el derecho, tanto en sí mismo como en su articulación con el orden y el desorden, son compartidas por los rituales. En su opinión, tanto el derecho como el ritual: (a) dependen de la regularidad y la predictibilidad, (b) dependen de la existencia de un orden en el mundo, y (c) respecto a ese orden, actúan en pos de articularlo, dispensarlo, recrearlo, imponerlo, y forzar su cumplimiento.

En base a estos elementos, Just concluirá que tanto "el derecho como el ritual comparten una profunda estructura derivada de servir a la tarea compartida de articular una visión del mundo que es ordenada moral y ontológicamente" 77 Es más, su lectura permite concluir que la diferencia entre ritual y derecho es producto de una reflexión de las instituciones occidentales, el colonialismo y de las divisiones y subdivisiones de la academia.

La propuesta de Just es interesante y, a la vez, radical. Ella invita a abandonar el presupuesto básico sobre el cual opera la ideología del centralismo legal, ésta es la idea de que existe una barrera definible entre rito y derecho. Así, "derecho oficial” y rito operan como esferas discretas, autónomas entre sí y claramente definibles. En reemplazo de esta imagen binaria, Just llama a "no ocuparse del derecho como un dominio discreto con un conjunto de características institucionales -cortes, jueces, cárceles, policía, etc.- sino a entenderlo como un conjunto de procesos sociales comprometidos con resolver disputas, resolver conflictos e imponer orden en el comportamiento"78. De acuerdo con esta propuesta, se advierte cómo dentro de estos procesos sociales los límites y distinciones entre ritualidad y derecho se vuelven absolutamente difusos y pasan a formar parte de la interlegalidad propuesta por Santos ${ }^{79}$.

\section{CRÍtítCAS}

La perspectiva del pluralismo legal no ha estado exenta de críticas. Tamanaha ${ }^{80}$ sintetiza los dos tipos de problemas más recurrentes en la literatura asociados al pluralismo legal: los analíticos y los instrumentales.

Los problemas analíticos dicen relación con la debilidad del pluralismo legal para definir qué es lo que se entiende por derecho. Dentro de este tipo de problemas, en primer lugar está el fenómeno de la diversidad conceptual. Diversos autores ${ }^{81}$, desde la plataforma del pluralismo legal, presentan sus propias definiciones de derecho, las que, sin ser del todo satisfactorias, no son coherentes entre ellas. Ello derivaría en una falta de entendimiento. Por lo mismo, "en la medida de que existan en competencia muchas

\footnotetext{
${ }^{77}$ Id. en p. 113. [La traducción es mía].

${ }^{78}$ Id. en p. 114. [La traducción es mía].

79 Santos, B., op. cit., n. 21.

${ }^{80}$ Tamanaha, B.Z., op. cit., n. 67.

81 Ver e.g., Id., Hoebel, E. A., Man in the primitive world; An introduction to anthropology. Harvard Univ. Press., 1954., Santos., op. cit., n. 21; Griffiths J., op. cit., n. 5, Galanter, M., op. cit., n. 11.
} 
versiones de lo que se entiende por derecho, la afirmación de que existe una pluralidad de derechos nos deja con una pluralidad de pluralismos legales" 82 .

El segundo problema del tipo analítico dice relación con la amplitud que se genera luego de que el derecho es posicionado fuera del Estado. La debilidad del pluralismo legal en alcanzar acuerdos sobre lo que es derecho, y su proposición de llevarlo afuera del dominio estatal generaría una confusión en la medida de que se hace muy difícil distinguir entre lo que es derecho y lo que es el resto del acontecer social. Como bien pregunta Merry: “¿Dónde terminamos de hablar de derecho y nos encontramos nosotros mismos simplemente describiendo la vida social?" 83 .

Los problemas instrumentales, a juicio de Tamanaha, son un función de los problemas analíticos. En su opinión, el principal inconveniente de este tipo es el hecho de que "se hace muy difícil reconceptualizar la relación derecho/sociedad si no hay acuerdo sobre qué es derecho y si las versiones adoptadas sobre lo que se entiende por derecho son incapaces de evitar que el derecho se trague a la vida social" 84 .

Como común denominador a los problemas analíticos e instrumentales, Tamanaha ${ }^{85}$ sostiene que el pluralismo legal ha pecado de ser esencialista en naturaleza. Ello por cuanto él ha entendido al derecho bajo la suposición de que "es una categoría fundamental, la cual puede ser identificable y descrita, o que es una noción esencial, la que puede ser internamente trabajada hasta generar una pura versión (de-contextualizada)" 86 .

\section{Conclusiones}

Los principales aportes del pluralismo legal radican en su problematización de la sinonimia entre Estado y derecho, y su constatación de la existencia de una pluralidad de órdenes normativos en la sociedad. Ello constituye una invitación a complejizar los estudios sociolegales en su comprensión de la relación entre derecho y orden social. El alejarse de la idea de que el Estado es el monopolizador de la ley, y el acercarse a estudiar otras formas de orden legal y las relaciones entre ellas y el Estado, es una invitación provocadora pero, al mismo tiempo, enriquecedora. Ello en la medida de que permite terminar con visiones simplistas, para destacar cómo existen formas de orden social que, estando fuera del Estado, se relacionan tanto entre sí como con el Estado, ya sea a través de dinámicas contradictorias, dialécticas, de mutua constitución, mímicas, de hibridización, entre otras.

Desde luego, dicha constatación y problematización no busca validar ningún sistema legal por sobre otro, proponer la eliminación del Estado, ni menos exige un

\footnotetext{
82 Tamanaha, B.Z., op. cit., n. 11, p. 297. [La traducción es mía].

${ }^{83}$ Merry, S. E., op. cit., n. 21, p. 878. [La traducción es mía].

84 Tamanaha, B.Z., op. cit., n. 67. p. 299. [La traducción es mía].

${ }^{85}$ Id., Tamanaha, B.Z., "The Folly of the "Social Scientific" Concept of Legal Pluralism", en Journal of Law and Society, vol. 20, $\mathrm{N}^{\circ}$ 2, 1993, 192-217.

${ }^{86} I d$., p. 299. [La traducción es mía].
} 
reconocimiento a todo y cada uno de los diversos órdenes normativos que comparten los espacios políticos. Simplemente, como lo muestra la genealogía del proyecto del pluralismo legal, este enfoque del derecho pretende develar cuáles son las relaciones de poder existentes entre quienes se atribuyen determinadas formas de organizar las acciones sociales el mundo, estén ellos o no dentro del aparataje estatal.

La ideología del centralismo legal, organizada bajo la idea liberal de Estado, presenta al Estado como garantía de equilibrio de poderes dentro de la sociedad. En este sentido, al ser el Estado la única fuente válida del derecho, todos quienes se atribuyan facultades normativas fuera de dicha institución quedan neutralizados o sometidos al poder normativo del Estado. Desde luego que ello puede resultar una idea tranquilizadora. Reconocerle validez normativa a la infinitud de organizaciones sociales como, por ejemplo, iglesias, grupos económicos, u otros grupos de interés, resulta preocupante y peligroso para la realización de cualquier proyecto de convivencia. Sin embargo, el pluralismo legal, lejos de defender los proyectos de autonomía normativa de dichas organizaciones, busca transparentar lo que el Estado oculta: el hecho de que las relaciones de dominados y dominantes no es una cuestión monopolizada por las normas jurídicas oficiales. Ante ello, el pluralismo legal debe constituirse como un proyecto que busca develar cómo diversos sistemas normativos que, enraizados en determinadas ideologías, reclaman para sí validez en su forma de ordenar el mundo.

A pesar de su interesante propuesta, como advierte Von Benda-Beckmann ${ }^{87}$, el pluralismo legal se ha estancado conceptualmente. Su mérito se ha reducido a rechazar al centralismo legal y constar una pluralidad jurídica. Ello, sin abordar de manera minuciosa la pregunta clave de qué es lo que se entiende por derecho u orden normativo ${ }^{88}$. Sin embargo, como advierte Tamanaha, la ausencia de una definición uniforme o satisfactoria no es una crítica propia del pluralismo legal, por el contrario, es aplicable a la doctrina del derecho en general, por lo mismo Hart, ante la pregunta de qué es derecho, ha afirmado que "pocas preguntas han sido [...] respondidas por pensadores serios de manera tan diversa, extraña, e inclusive paradójica" 89 . Ahora, el problema relativo a los límites entre lo que es o no derecho resulta problemático en la medida de que puede derivar en un reemplazo de la ideología del centralismo legal por una ideología del fetichismo legal, que reduzca a derecho o categorías jurídicas todo fenómeno social. Ello, sin duda, requiere más reflexión, de lo contrario el pluralismo legal jamás será el "concepto clave de una visión postmoderna del derecho" 90 , ni mucho menos "el tema central de la reconceptualización de la relación entre derecho y sociedad"91.

${ }^{87}$ Von Benda-Beckmann, F., "Comment on Merry", en Law and Society Review. Vol. 22, N 5, 1988, pp. 897-902. Tamanaha, B. Z., op. cit., n. 67.

${ }^{88} \mathrm{Id}$.

${ }^{89}$ Hart, H. L. A., The Concept of Law, Clarendon Press, Oxford, 1961, p. 1. [La traducción es mía].

${ }^{90}$ Santos, B., op. cit., n. 21, p. 297. [La traducción es mía].

${ }^{91}$ Merry, S. E., op. cit., n. 10, p. 869. [La traducción es mía]. 


\section{BIBLIOGRAFÍA}

Althusser, L., Ideología y apparatus ideological del Estado, Quinto Sol, México, D. F., 1985.

Banner, S., "Preparing to be Colonized: Land Tenure and Legal Strategy in Nineteenth-Century Hawaii”, en Law E Society Review, vol. 39, N², Junio 2005, pp. 273-314.

Barthes, R., Mitologías, Siglo XXI, Buenos Aires, Argentina, 2002.

Berman, P. S., "The New Legal Pluralism", en Annual Review of Law and Social Science, vol. 5, 2009, pp. 225-242.

Bernard, B., "Interpretación postmoderna del pluralismo legal como fundamento para una nueva Teoría del Derecho”, en Frónesis, vol. 13, N 1, 2006, pp. 1315-6268.

Boelens, R.A. (ed.)., Pluralismo Legal, Reformas Hidricas y Políticas de Reconocimiento. Walir, 2007.

Bohannan, P., Justice and Judgment Among the Tiv, International African Institute by Oxford University Press, London, New York, 1957.

Burke-White, W. W., "International Legal Pluralism”, en Michigan Journal of International Law. vol. 25, N 4, 2004, pp. 963-979.

Carmona, C., "Derecho y Violencia: Reescrituras en torno al pluralismo jurídico", en Revista de derecho (Valdivia), vol. 22, N², Diciembre 2009, pp. 9-26.

Castro, M., "Agua, derechos y cultura en los Andes del norte de Chile. Un enfoque desde la antropología jurídica”, en Chungara, vol. 29, N 1, 1997, pp. 63-80.

Castro, M. (ed.), Actas del XII Congreso Internacional. Derecho Consuetudinario y Pluralismo Legal: Desafíos del Tercer Milenio, Universidad de Chile, Santiago, Chile, 2000.

Chase, O. G., Law, Culture, and Ritual: Disputing Systems in Cross-Cultural Context, New York University Press, New York, 2005.

Diamond, S., "The Rule of Law versus the Order of Custom", en Black D. y Mileski, M. (eds.), The Social Organization of Law, Seminar Press, New York, 1973. pp. 318-344.

Evans-Pritchard, E. E., Witchcraft, Oracles and Magic Among the Azande, Clarendon Press, Oxford, 1937.

FitzPatrick P., "Law and societies”, en Osgoode Hall, Law Journal, vol. 22. ํ 1, 1984, p. 297. FitzPatrick, P., Law and State in New Guinea, Academic Press, London, 1981.

Foucault, M., Nacimiento de la Biopolitica: Curso en El College de France 1978-1979, Fondo de Cultura Económica, Argentina, 2007.

Galanter, M., "Justice in Many Rooms: Courts, Private Ordering and Indigenous Law", en Journal of Legal Pluralism, vol. 19, $\mathrm{N}^{\circ} 1,1981$, pp. 1-47.

García, L., "Pluralismo Legal y Derecho Indígena", en Frónesis, vol. 13, Nº.1, 2006, pp. 1315-6268.

Geertz, C., Local knowledge: Further essays in interpretive anthropology, Basic Books, New York, 1983.

Glickman, M., The judicial process among the Barotse of Northern Rhodesia, Manchester U.P., Manchester, 1967.

Griffiths J., "What is legal pluralism?", en Journal of Legal Pluralism and Unofficial Law, vol. 24, 1986, pp. 1-55.

Hart, H. L. A., The Concept of Law, Clarendon Press, Oxford, 1961.

Harvey, D., "The Marxian Theory of the State", en Antipode, vol. 8, N², 1976, pp. 80-89.

Hoebel, E. A., Man in the primitive world; An introduction to anthropology. Harvard Univ. Press., 1954.

Hooker, M. B., Legal Pluralism: An Introduction to Colonial and Neo-Colonial Laws. Clarendon Press, Oxford, 1975.

Just, P., "Law, Ritual and Order", en Benda-Beckmann, K.V. y Pirie, F. (eds.), Order and disorder: Anthropological perspectives, Berg Hahn Books, New York, 2007.

Lansing, J. S., Perfect Order: Recognizing Complexity in Bali, Princeton University Press, Princeton, N. J., 2006. 
Llewellyn, K. N. y Hoebel, E. A., The Cheyenne Way: Conflict and Case Law in Primitive Jurisprudence, University of Oklahoma Press, Norman, 1941.

Malinowski, B., Crime and Custom in Savage Society, Harcourt, Brace and Co., New York, 1932.

Marshall, J., The meat fight. Watertown, M A: Documentary Educational Resources, 2007.

Merry, S. E., "Colonial and Postcolonial Law", en Sarat, A. (ed.), Blackwell Companion to Law and Society. Blackwell Publishing, Oxford, 2004. pp. 569-588.

Merry, S. E., "Anthropology and International Law", en Annual Review of Anthropology, vol. 35, 1996, pp. 99-116.

Merry, S. E., "Legal Pluralism”, en Law E Society Review. vol. 22, N 5, 1988, pp. 869-892.

Michaels, R., "Global Legal Pluralism”, en Annual Review of Law E Social Science, vol. 5, 2009, pp. 243-62.

Moore, S. F., Law and Anthropology: A Reader, Blackwell, Malden, MA. 2005.

Moore, S. F., "Certainties Undone: Fifty Turbulent Years of Legal Anthropology, 1949-1999", en Journal of the Royal Anthropological Institute, vol. 7, N 1, 2001, pp. 98-116.

Moore S. F., "Law and social change: the semi-autonomous social field as an appropriate subject of study", en Law E Society Review, vol. 7, N 4, 1973, pp. 719-746.

Perrin, J. F., "La autonomía de la voluntad y el pluralismo jurídico en nuestros días", en Sociologías, $\mathrm{N}^{\circ} 13,2005$, pp. 162-178.

Pospisil, L., The Anthropology of Law: A Comparative Theory of Law. Harper and Row, New York, 1971.

Rodman, W. L., "A Law Unto Themselves": Legal Innovation in Ambae, Vanuatu”, en American Ethnologist, vol. 12, $\mathrm{N}^{\circ}$ 4, noviembre 1985), pp. 603-624.

Santos, B. S., Law: A Map of Misreading. "Toward a Postmodern Conception of Law”, en Journal of Law and Society, vol. 14, $\mathrm{N}^{\circ} 3$, pp. 279-302.

Schiff, P., "The New Legal Pluralism”, en Annual Review of Law and Social Science, vol. 5, 2009, pp. 225-242.

Silbey S. S. y Seron, C., "Profession, Science, and Culture: An Emergent Canon of Law and Society Research" en Sarat, A. (ed.), Blackwell Companion to Law and Society. Blackwell Publishing, Oxford, 2004. pp. 30-59.

SINGER, A., Witchcraft among the Azande, Granada Television International (Filmmakers Library), 1982.

Tamanaha, B. Z., "Understanding Legal Pluralism: Past to Present, Local to Global”, en The Sydney Law Review, vol. 30, $\mathrm{N}^{\circ}$ 3, 2008, pp. 375-411.

Tamanaha, B. Z., "A Non-Essentialist Version of Legal Pluralism”, en Journal of Law and Society, vol. 27, $\mathrm{N}^{\circ}$ 2, 2000, pp. 296-321.

Tamanaha, B. Z., "The Folly of the "Social Scientific" Concept of Legal Pluralism”, en Journal of Law and Society, vol. 20, $\mathrm{N}^{\circ} 2,1993,192-217$.

Teubner, G., "The Two Faces of Janus: Rethinking Legal Pluralism”, en Cardozo Law Review, 1992, pp. 1443-62.

VANDERLINDEN J., "Return to legal pluralism: twenty years later", en Journal of Legal Pluralism and Unofficial Law; $\mathrm{N}^{\circ} 28,1989$, pp. 149-157.

Von Benda-Beckmann, F. V., "Comment on Merry", en Law and Society Review. vol. 22, $\mathrm{N}^{\circ}$ 5, 1988, pp. 897-902.

Von Benda-Beckmann, F. V., "Anthropology and Comparative Law” en Benda-Beckmann, K. V. y Strijbosch, F. (eds.), Anthropology of Law in the Netherlands, Foris, Dordrecht, 1986.

ŽižeK, S., "El Espectro de la ideología”, en Žižek, S. (ed.), Ideología, un mapa de la cuestión, Fondo de Cultura Económica, Buenos Aires, Argentina, 2003, pp. 7-42. 
